УДК 517.55

\title{
Holomorphic Extension of Continuous Functions Along Finite Families of Complex Lines in a Ball
}

\author{
Alexander M. Kytmanov* \\ Simona G. Myslivets ${ }^{\dagger}$ \\ Institute of Mathematics and Computer Science \\ Siberian Federal University \\ Svobodny, 79, Krasnoyarsk, 660041
}

Russia

Received 10.06.2015, received in revised form 01.07.2015, accepted 25.07.2015

In this paper we consider continuous functions given on the boundary of a ball $B$ of $\mathbb{C}^{n}, n>1$, and having one-dimensional property of holomorphic extension along the families of complex lines, passing through finite number of points of $B$. We prove the existence of holomorphic extension of such functions in the ball $B$.

Keywords: holomorphic extension, Poisson kernel, complex lines.

DOI: $10.17516 / 1997-1397-2015-8-3-291-302$

\section{Introduction}

This paper presents some results related to the holomorphic extension of functions $f$, defined on the boundary of a ball $B \subset \mathbb{C}^{n}, n>1$, in this ball. We deal with a functions with the one-dimensional holomorphic extension property along the complex lines.

The first result related to our subject was received by M.L.Agranovsky and R.E.Valsky in [1], who studied the functions with a one-dimensional holomorphic continuation property on the boundary of a ball. The proof was based on the automorphism group properties of a sphere.

E.L.Stout in [2] used complex Radon transformation to generalize the Agranovsky and Valsky theorem for an arbitrary bounded domain with a smooth boundary. An alternative proof of the Stout theorem was obtained by A.M.Kytmanov in [3] by applying the Bochner-Martinelli integral. The idea of using the integral representations (Bochner-Martinelli, Cauchy-Fantappiè, multidimensional logarithmic residue) has been useful in the study the functions with onedimensional holomorphic continuation property (see review [4]).

The problem of finding the different families of complex lines, sufficient for holomorphic extension was posed in [5]. Clearly, the family of complex lines passing through one point is not enough. As shown in [6], the family of complex lines passing through a finite number of points also, generally speaking, is not sufficient.

In [6] we proved that the family of complex lines crossing the germ of generic manifold $\gamma$, is sufficient for the holomorphic extension. In [7] we consider a family of complex lines passing through the germ of a complex hypersurface. In particular, $\mathbb{C}^{2}$, it can be any real analytic curve. Various other families are given in [8-11]. We note here the work [9,11], where it is shown that a family of complex lines passing through somehow located a finite number of points is sufficient for holomorphic extension. But it is approved only for real-analytic or infinitely differentiable functions defined on the boundary. Then in $\mathbb{C}^{2}$ Agranovsky and Globevnik for real-analytic

\footnotetext{
*akytmanov@sfu-kras.ru

†asmyslivets@sfu-kras.ru

(c) Siberian Federal University. All rights reserved
} 
functions defined on the boundary shown that it is enough two points lying in the closure of the ball. The Globevnik example shows that for continuous functions on the boundary of two points is not enough for holomorphic extension.

In [12-15] it is shown that for a class of continuous functions given on the boundary of a ball the family of complex lines passing through finite points in the ball is sufficient. Baracco was the first to prove this result which earlier explicitly conjectured in [9]. Globevnik [13] gave an alternative proof, even the case when the vertices lie outside of the ball. Authors in [14,15] for proof use the Poisson kernel and the automorphisms of a ball.

Those results it is given by completely different methods.

In this paper we give a new proof of results from $[14,15]$ without use the automorphisms of a ball.

\section{Invariant Poisson kernel of a ball}

Let $B=\left\{z \in \mathbb{C}^{n}:|z|<1\right\}$ be the unit ball in $\mathbb{C}^{n}, n>1$, centered at the origin and let $S=\partial B$ be a boundary of the ball. Consider the invariant Poisson kernel [17, p. 48]

$$
P(z, \zeta)=c_{n} \frac{\left(1-|z|^{2}\right)^{n}}{|1-\langle z, \bar{\zeta}\rangle|^{2 n}}=c_{n} \frac{(1-\langle z, \bar{z}\rangle)^{n}}{(1-\langle z, \bar{\zeta}\rangle)^{n}(1-\langle\zeta, \bar{z}\rangle)^{n}},
$$

where $c_{n}=\frac{(n-1) !}{2 \pi^{n}},\langle z, \zeta\rangle=z_{1} \zeta_{1}+\cdots+z_{n} \zeta_{n}$.

If the function $f(z)$ is $\mathcal{M}$-harmonic in $B$ and continuous on $\bar{B}$, then we have the integral representation

$$
F(z)=\int_{S} f(\zeta) P(z, \zeta) d \sigma(\zeta)
$$

where

$$
d \sigma(\zeta)=\frac{2}{i^{n}} \sum_{k=1}^{n}(-1)^{k-1} \bar{\zeta}_{k} d \bar{\zeta}[k] \wedge d \zeta
$$

is Lebesgue measure on $S, d \zeta=d \zeta_{1} \wedge \cdots \wedge d \zeta_{n}, d \bar{\zeta}[k]=d \bar{\zeta}_{1} \wedge \cdots \wedge d \bar{\zeta}_{k-1} \wedge d \bar{\zeta}_{k+1} \wedge \cdots \wedge d \bar{\zeta}_{n}$. The boundary values of function $F(z)$ coincides with $f(\zeta)$, i.e., $\left.F(z)\right|_{S}=f(\zeta)$. Recall that $\mathcal{M}$-harmonic function satisfies the Laplace equation [17, p. 55-56]

$$
\widetilde{\triangle} F(z)=0
$$

where

$$
\widetilde{\triangle} F(z)=4\left(1-|z|^{2}\right) \sum_{j, k=1}^{n}\left(\delta_{j k}-z_{j} \bar{z}_{k}\right) \frac{\partial^{2} F(z)}{\partial z_{j} \partial \bar{z}_{k}}
$$

and $\delta_{j k}$ is Kronecker symbol. The functions holomorphic in the ball $B$ are $\mathcal{M}$-harmonic. In particular, the formula (1) is an integral representation for holomorphic functions.

Consider the complex line of the form

$$
l_{z, b}=\left\{\zeta \in \mathbb{C}^{n}: \zeta=z+b t, t \in \mathbb{C}\right\},
$$

where $z \in \mathbb{C}^{n}, b \in \mathbb{C P}^{n-1}$. Complex lines passing through two points $a$ and $c$ is denoted by $l_{a, c}$.

We will say that a function $f \in \mathcal{C}(S)$ has the one-dimensional holomorphic extension property along the complex line $l_{z, b}$, if $S \cap l_{z, b} \neq \varnothing$ and there exists a function $F_{l_{z, b}}$ with the following properties:

1) $F_{l_{z, b}} \in \mathcal{C}\left(\bar{B} \cap l_{z, b}\right)$, 
2) $F_{l_{z, b}}=f$ on the set $S \cap l_{z, b}$,

3) function $F_{l_{z, b}}$ is holomorphic at the interior (with respect to the topology of $l_{z, b}$ ) points of set $\bar{B} \cap l_{z, b}$.

Let $\Gamma$ is a set in $\mathbb{C}^{n}$. Denote by $\mathfrak{L}_{\Gamma}$ the set of all complex lines $l_{z, b}$ such that $z \in \Gamma$, and $b \in \mathbb{C P}^{n-1}$, i.e., the set of all complex lines passing through $z \in \Gamma$.

We will say that a function $f \in \mathcal{C}(S)$ has the one-dimensional holomorphic extension property along the family $\mathfrak{L}_{\Gamma}$, if it has the one-dimensional holomorphic extension property along any complex line $l_{z, b} \in \mathfrak{L}_{\Gamma}$.

We will say, that set $\mathfrak{L}_{\Gamma}$ is sufficient for holomorphic extension, if the function $f \in \mathcal{C}(S)$ has the one-dimensional holomorphic extension property along all complex lines of the family $\mathfrak{L}_{\Gamma}$, and then the function $f$ extends holomorphically to $B$ (i.e., $f$ is a $C R$-function on $S$ ). The different families of sufficient sets were considered by Agranovsky, Valsky, Stout, Globevnik, Baracco and the authors of this article.

\section{Complexification of the Poisson kernel and its applications}

Consider the kernel of the form

$$
Q(z, w, \zeta)=c_{n} \frac{(1-\langle z, w\rangle)^{n}}{(1-\langle z, \bar{\zeta}\rangle)^{n}(1-\langle\zeta, w\rangle)^{n}} .
$$

Obviously, that $P(z, \zeta)=Q(z, \bar{z}, \zeta)$. We introduce the function

$$
\Phi(z, w)=\int_{S} f(\zeta) Q(z, w, \zeta) d \sigma(\zeta) .
$$

This function is holomorphic in the variables $(z, w)$ in $B \times B \subset \mathbb{C}^{2 n}$, because the denominator in the kernel (4) does not vanish, when $\zeta \in S$ and $z, w \in B$. Note that $\Phi(z, \bar{z})=F(z)$, and the derivatives

$$
\left.\frac{\partial^{\alpha+\beta} \Phi}{\partial z^{\alpha} \partial w^{\beta}}\right|_{w=\bar{z}}=\frac{\partial^{\alpha+\beta} F}{\partial z^{\alpha} \partial \bar{z}^{\beta}}
$$

where

$$
\frac{\partial^{\alpha+\beta} \Phi}{\partial z^{\alpha} \partial w^{\beta}}=\frac{\partial^{\|\alpha\|+\|\beta\|} \Phi}{\partial z_{1}^{\alpha_{1}} \cdots \partial z_{n}^{\alpha_{n}} \partial w_{1}^{\beta_{1}} \cdots \partial w_{n}^{\beta_{n}}}
$$

and $\alpha=\left(\alpha_{1}, \ldots, \alpha_{n}\right), \beta=\left(\beta_{1}, \ldots, \beta_{n}\right)$ are multi-indices. We denote $\|\alpha\|=\alpha_{1}+\cdots+\alpha_{n}$, $\|\beta\|=\beta_{1}+\cdots+\beta_{n}$.

Theorem 1. Let a function $f(\zeta) \in \mathcal{C}(S)$ have the one-dimensional holomorphic extension property along the family $\mathfrak{L}_{\{0\}}$, then for the integral

$$
\Phi(z, w)=\int_{S} f(\zeta) Q(z, w, \zeta) d \sigma(\zeta)
$$

true the properties $\Phi(0, w)=$ const and the derivatives $\frac{\partial^{\alpha} \Phi(0, w)}{\partial z^{\alpha}}$ are polynomials in $w$ of degree not higher than $\|\alpha\|$.

Proof. Consider the derivative

$$
\frac{\partial^{\alpha+\beta}}{\partial z^{\alpha} \partial w^{\beta}}\left(\frac{1}{(1-\langle z, \bar{\zeta}\rangle)^{n}(1-\langle\zeta, w\rangle)^{n}}\right)=\frac{C_{\alpha, \beta} \bar{\zeta}^{\alpha} \zeta^{\beta}}{(1-\langle z, \bar{\zeta}\rangle)^{n+\|\alpha\|}(1-\langle\zeta, w\rangle)^{n+\|\beta\|}},
$$


where $\zeta^{\alpha}=\zeta_{1}^{\alpha_{1}} \cdots \zeta_{n}^{\alpha_{n}}, \bar{\zeta}^{\alpha}=\bar{\zeta}_{1}^{\alpha_{1}} \cdots \bar{\zeta}_{n}^{\alpha_{n}}$, and $C_{\alpha, \beta}$ are some constants.

It is easy to show that the derivative of $\frac{\partial^{\alpha+\beta} Q(0, w)}{\partial z^{\alpha} \partial w^{\beta}}$ is the sum of terms of a form $\frac{C_{\alpha, \beta, \gamma, \delta} \bar{\zeta}^{\alpha-\gamma^{\prime}} \zeta^{\beta-\gamma^{\prime \prime}} w^{\delta}}{(1-\langle\zeta, w\rangle)^{n+\|\beta\|-\left\|\gamma^{\prime \prime}\right\|}}$ at $\|\delta\| \leqslant n,\left\|\gamma^{\prime \prime}\right\| \leqslant\left\|\gamma^{\prime}\right\|$, and $\alpha \geqslant \gamma^{\prime}, \beta \geqslant \gamma^{\prime \prime}$. Therefore, the derivative $\frac{\partial^{\alpha+\beta} \Phi(0, w)}{\partial z^{\alpha} \partial w^{\beta}}$ is a linear combination of integrals

$$
\int_{S} f(\zeta) \frac{\bar{\zeta}^{\alpha-\gamma^{\prime}} \zeta^{\beta-\gamma^{\prime \prime}}}{(1-\langle\zeta, w\rangle)^{n+\|\beta\|-\left\|\gamma^{\prime \prime}\right\|}} d \sigma(\zeta)
$$

The form $d \sigma(\zeta)$ in variables $b$ and $t$, where $\zeta=b t, t \in \mathbb{C}$, was calculated in ( [4])

$$
d \sigma(b t)=\frac{2}{i^{n}}|t|^{2 n-2} \bar{t} d t \wedge\left(\sum_{k=1}^{n}(-1)^{k-1} b_{k} d b[k]\right) \wedge\left(\sum_{k=1}^{n}(-1)^{k-1} \bar{b}_{k} d \bar{b}[k]\right) .
$$

Since on the sphere $S$ the equality $1=|\zeta|=|b t|$ holds, then $|t|=\frac{1}{|b|}$ and $\bar{t}=\frac{1}{t|b|^{2}}$. So

$$
d \sigma(b t)=\frac{2}{i^{n}} \frac{1}{t|b|^{2 n}} d t \wedge\left(\sum_{k=1}^{n}(-1)^{k-1} b_{k} d b[k]\right) \wedge\left(\sum_{k=1}^{n}(-1)^{k-1} \bar{b}_{k} d \bar{b}[k]\right)=\lambda(b) \wedge \frac{d t}{t} .
$$

From Fubini's theorem we obtain

$$
\begin{aligned}
\int_{S} f(\zeta) \frac{\bar{\zeta}^{\alpha-\gamma^{\prime}} \zeta^{\beta-\gamma^{\prime \prime}}}{(1-\langle\zeta, w\rangle)^{n+\|\beta\|-\left\|\gamma^{\prime \prime}\right\|}} d \sigma(\zeta)= \\
=\int_{\mathbb{C P}^{n-1}} \lambda(b) \int_{S \cap l_{0, b}} f(b t) \frac{\bar{t}^{\|\alpha\|-\left\|\gamma^{\prime}\right\|} t^{\|\beta\|-\left\|\gamma^{\prime \prime}\right\|}}{t(1-t\langle b, w\rangle)^{n+\|\beta\|-\left\|\gamma^{\prime \prime}\right\|}} d t= \\
=\int_{\mathbb{C P}^{n-1}} \lambda(b) \int_{S \cap l_{0, b}} f(b t) \frac{t^{\|\beta\|-\left\|\gamma^{\prime \prime}\right\|}}{t^{\|\alpha\|-\left\|\gamma^{\prime}\right\|+1}(1-t\langle b, w\rangle)^{n+\|\beta\|-\left\|\gamma^{\prime \prime}\right\|}} d t=0
\end{aligned}
$$

if $\|\beta\|>\|\alpha\|$ (then $\left.\|\beta\|-\left\|\gamma^{\prime \prime}\right\|>\|\alpha\|-\left\|\gamma^{\prime}\right\|\right)$, and the function $\frac{1}{1-t\langle b, w\rangle}$ is holomorphic in the closed disk $|t|<\frac{1}{|b|}$, such that $\bar{B} \cap l_{0, b}$. Hence by virtue of $(6)$

$$
\frac{\partial^{\alpha+\beta} \Phi(0, w)}{\partial z^{\alpha} \partial w^{\beta}}=0
$$

at $\|\beta\|>\|\alpha\|$.

Therefore, by the Taylor formula for the function $\Phi(z, w)$ at the point $(0,0)$ we get that $\Phi(0, w)=$ const and the derivatives $\frac{\partial^{\alpha} \Phi(0, w)}{\partial z^{\alpha}}$ are polynomials in $w$ of degree not higher than $\|\alpha\|$.

Corollary 1. Under the hypotheses of Theorem 1 the equality

$$
\frac{\partial^{\alpha+\beta} F(0,0)}{\partial z^{\alpha} \partial \bar{z}^{\beta}}=0
$$

holds if $\|\beta\|>\|\alpha\|$. 
Proof. Substituting in Eq. (7) $w=\bar{z}$ and using Eq. (5), we obtain the desired result.

Theorem 2. Let the function $f(\zeta) \in \mathcal{C}(S)$ have the one-dimensional holomorphic extension property along the family $\mathfrak{L}_{\{a\}}, a \in B, a \neq 0$, then $\Phi(a, w)=$ const and the derivatives $\frac{\partial^{\alpha} \Phi(a, w)}{\partial z^{\alpha}}$ are polynomials in $w$ of degree not higher than $\|\alpha\|$.

Proof. Consider the function $\Phi(z, w)$ and a complex line $l_{a, b}$. Since

$$
\frac{\partial^{\beta} \Phi(z, w)}{\partial w^{\beta}}=\int_{S} f(\zeta) \frac{\partial^{\beta} Q(z, w, \zeta)}{\partial w^{\beta}} d \sigma(\zeta)
$$

we calculate

$$
\begin{aligned}
& \frac{\partial^{\beta} Q(z, w, \zeta)}{\partial w^{\beta}}=\frac{\partial^{\beta}}{\partial w^{\beta}}\left((1-\langle z, w\rangle)^{n} \cdot \frac{1}{(1-\langle z, \bar{\zeta}\rangle)^{n}(1-\langle\zeta, w\rangle)^{n}}\right)= \\
& =c_{n} \sum_{0 \leqslant \gamma \leqslant \beta} b_{\gamma} \frac{\partial^{\beta-\gamma}}{\partial w^{\beta-\gamma}}\left(\frac{1}{(1-\langle z, \bar{\zeta}\rangle)^{n}(1-\langle\zeta, w\rangle)^{n}}\right) \frac{\partial^{\gamma}}{\partial w^{\gamma}}(1-\langle z, w\rangle)^{n}= \\
& =c_{n} \sum_{0 \leqslant \gamma \leqslant \beta} \frac{b_{\gamma} c_{\beta}(-1)^{\|\gamma\|} \zeta^{\beta-\gamma} n(n-1) \cdot \ldots \cdot(n-\|\gamma\|)(1-\langle z, w\rangle)^{n-\|\gamma\|} z^{\gamma}}{(1-\langle z, \bar{\zeta}\rangle)^{n}(1-\langle\zeta, w\rangle)^{n+\|\beta-\gamma\|}}= \\
& =c_{n} \sum_{0 \leqslant \gamma \leqslant \beta} d_{\beta \gamma} \frac{\zeta^{\beta-\gamma}(1-\langle\zeta, w\rangle)^{n-\|\gamma\|} z^{\gamma}}{(1-\langle z, \bar{\zeta}\rangle)^{n}(1-\langle\zeta, w\rangle)^{n+\|\beta-\gamma\|}}
\end{aligned}
$$

Let the multi-index $\beta^{\prime}=\left(\beta_{1}, \ldots, \beta_{n-1}\right)$ such that $\left\|\beta^{\prime}\right\|=\beta_{1}+\cdots+\beta_{n-1}>0$. Using a unitary transform, we will transfer the point $a$ in the point $(0, \ldots, 0,|a|)$. Under this transform the kind of the form $d \sigma$ does not change (see [16, Lemma 2.7]). We get

$$
\frac{\partial^{\beta} Q(a, w, a+b t)}{\partial w^{\beta}}=c_{n} \sum_{0 \leqslant \gamma \leqslant \beta} d_{\beta \gamma} \frac{(a+b t)^{\beta-\gamma}(1-\langle a, w\rangle)^{n-\|\gamma\|} a^{\gamma}}{(1-\langle a, \bar{a}+\bar{b} \bar{t}\rangle)^{n}(1-\langle a+b t, w\rangle)^{n+\|\beta-\gamma\|}}
$$

at $\zeta=a+b t$ and $z=a$. The expression $(a+b t)^{\beta-\gamma}$ means $\left(a_{1}+b_{1} t\right)^{\beta_{1}-\gamma_{1}} \cdots\left(a_{n}+b_{n} t\right)^{\beta_{n}-\gamma_{n}}$.

Since $B \cap l_{a, b}$ is a circle

$$
K=\left\{t \in \mathbb{C}:\left|t+\frac{\langle a, \bar{b}\rangle}{|b|^{2}}\right|^{2} \leqslant \frac{\left(1-|a|^{2}\right)|b|^{2}+|\langle a, b\rangle|^{2}}{|b|^{4}}\right\}
$$

with center at the point $t_{0}=-\frac{\langle a, \bar{b}\rangle}{|b|^{2}}$, then we consider the restriction of the form $d \sigma$ on the boundary of this circle, provided that $|a+b t|=1$ :

$$
\begin{aligned}
d \sigma=\sum_{k=1}^{n}(-1)^{k-1} \bar{\zeta}_{k} d \bar{\zeta}[k] \wedge d \zeta & = \\
& =\sum_{k=1}^{n}(-1)^{k-1}\left(\bar{a}_{k}+\bar{b}_{k} \bar{t}\right) \bar{t}^{n-1} t^{n-1} d \bar{b}[k] \wedge d t \wedge \sum_{j=1}^{n}(-1)^{j-1} b_{j} d b[j] .
\end{aligned}
$$


Since $\bar{t}=\frac{1-t\langle\bar{a}, b\rangle-|a|^{2}}{\langle a, \bar{b}\rangle+t|b|^{2}}$ then

$$
1-\langle a, \bar{a}+\bar{b} \bar{t}\rangle=\frac{t\left(\left(1-|a|^{2}\right)|b|^{2}+|\langle a, \bar{b}\rangle|^{2}\right)}{\langle a, \bar{b}\rangle+t|b|^{2}} .
$$

Therefore

$$
\begin{aligned}
\frac{\partial^{\beta} \Phi(a, w)}{\partial w^{\beta}}=c_{n} \int_{S} f(\zeta) \sum_{0 \leqslant \gamma \leqslant \beta} & d_{\beta \gamma} \frac{(a+b t)^{\beta-\gamma}(1-\langle a, w\rangle)^{n-\|\gamma\|} a^{\gamma}}{(1-\langle a, \bar{a}+\bar{b} \bar{t}\rangle)^{n}(1-\langle a+b t, w\rangle)^{n+\|\beta-\gamma\|}} \times \\
& \times \sum_{k=1}^{n}(-1)^{k-1}\left(\bar{a}_{k}+\bar{b}_{k} \bar{t}\right) \bar{t}^{n-1} t^{n-1} d \bar{b}[k] \wedge d t \wedge \sum_{j=1}^{n}(-1)^{j-1} b_{j} d b[j] .
\end{aligned}
$$

Since $\bar{t}=\frac{1-t\langle\bar{a}, b\rangle-|a|^{2}}{\langle a, \bar{b}\rangle+t|b|^{2}}$ is performed on the boundary of the circle $K$, then power of denominator $\langle a, b\rangle+t|b|^{2}$ goes to numerator of all fraction and it reduced with denominator of $\bar{t}$.

From the form of the point $a$ we get the expression $(a+b t)^{\beta-\gamma}$ contains a factor $t$ in the positive degree. By assumption of theorem $\frac{\partial^{\beta} \Phi(a, w)}{\partial w^{\beta}}=0$ and $\Phi(a, w)=$ const.

Similar it is proved that the derivatives $\left.\frac{\partial^{\alpha+\beta} \Phi(z, w)}{\partial z^{\alpha} \partial w^{\beta}}\right|_{z=a}=0$ if $\|\beta\|>\|\alpha\|$ and therefore the derivatives $\frac{\partial^{\alpha} \Phi(a, w)}{\partial z^{\alpha}}$ are polynomials on $w$ of degree not higher than $\|\alpha\|$.

\section{Decomposition of the Poisson kernel}

We represent the function $\Phi(z, w)$ as a sum of homogeneous polynomials in $z$ and $w$. We decompose $Q(z, w, \zeta)$ by powers of $\langle z, \bar{\zeta}\rangle,\langle\zeta, w\rangle$. Since

$$
\begin{aligned}
& \frac{1}{(1-\langle z, \bar{\zeta}\rangle)^{n}}=\sum_{k=0}^{\infty} C_{n+k-1}^{k}\langle z, \bar{\zeta}\rangle^{k}, \\
& \frac{1}{(1-\langle\zeta, w\rangle)^{n}}=\sum_{l=0}^{\infty} C_{n+l-1}^{l}\langle\zeta, w\rangle^{k}
\end{aligned}
$$

(considered the series converge absolutely for $\zeta \in S, z, w \in B$, and uniformly on $S \times K$, where $K$ is arbitrary compact set in $B \times B)$, then

$$
Q(z, w, \zeta)=c_{n}(1-\langle z, w\rangle)^{n} \sum_{k=0}^{\infty} \sum_{l=0}^{\infty} C_{n+k-1}^{k} C_{n+l-1}^{l} \int_{S} f(\zeta)\langle z, \bar{\zeta}\rangle^{k}\langle\zeta, w\rangle^{l} d \sigma(\zeta) .
$$

The integral $\int_{S} f(\zeta)\langle z, \bar{\zeta}\rangle^{k}\langle\zeta, w\rangle^{l} d \sigma(\zeta)$ is a homogeneous polynomial of degree of homogeneity $k$ in $z$ and $l$ in $w$. Multiplying the sum of Eq. (8) by a factor of $(1-\langle z, w\rangle)^{n}$ and regrouping the terms we find that

$$
\Phi(z, w)=\sum_{k, l=0}^{\infty} P_{k, l}(z, w)
$$


where $P_{k, l}(z, w)$ are homogeneous holomorphic polynomials of degree of homogeneity of $k$ in $z$ and $l$ in $w$. The double series converges absolutely in $B \times B$ and uniformly on any compact set in $B \times B$.

Theorem 3. Let the function $f(\zeta) \in \mathcal{C}(S)$, the point $a \in B$ and let the function $\Phi(z, w)$ satisfy the conditions: $\Phi(0, w)=$ const, $\Phi(a, w)=$ const, $\frac{\partial^{\alpha} \Phi(0, w)}{\partial z^{\alpha}}$ are polynomials in $w$ of degree not higher than $\|\alpha\|$, then for any fixed $z$, belonging to the complex line $l_{0, a}=\left\{z \in \mathbb{C}^{n}: z=\right.$ at, $|t|<1\}$, function $\Phi(z, w)=$ const in $w$, i.e., $\frac{\partial^{\beta} \Phi(z, w)}{\partial w^{\beta}}=0$ at $\|\beta\|>0$.

Proof. We represent the function $\Phi(z, w)$ in the form (9):

$$
\Phi(z, w)=\sum_{k, l=0}^{\infty} P_{k, l}(z, w) .
$$

By the hypothesis expansion of (9) takes the form

$$
\Phi(z, w)=\sum_{k \geqslant l \geqslant 0} P_{k, l}(z, w),
$$

since the derivatives $\frac{\partial^{\alpha+\beta} \Phi(0,0)}{\partial z^{\alpha} \partial w^{\beta}}=0$ at $\|\beta\|>\|\alpha\|$.

We introduce the functions $\Phi_{k}(z, w)=\sum_{l=k}^{\infty} P_{k, l}(z, w)$, then

$$
\Phi(z, w)=\sum_{k=0}^{\infty} \Phi_{k}(z, w) .
$$

Considered series converges absolutely in $B \times B$, uniformly on compact subsets of $B \times B$, since double series (9) converges absolutely in $B \times B$ and uniformly on compact subsets of $B \times B$, and the series (10) is repeated series of series (9).

From the form of the series $\Phi_{k}(z, w)$ we get that $\Phi_{k}(t z, w)=t^{k} \Phi_{k}(z, w)$ for every $t \in \mathbb{C}$. By the hypothesis

$$
\Phi(0, w)=\Phi_{0}(0, w)=\sum_{l=0}^{\infty} P_{0, l}(0, w)=\mathrm{const}
$$

and

$$
\Phi(a, w)=\sum_{k=0}^{\infty} \Phi_{k}(a, w)=\text { const } .
$$

Consider

$$
\Phi(a t, w)=\sum_{k=0}^{\infty} t^{k} \Phi_{k}(a, w) .
$$

We calculate

$$
\frac{d^{m}}{d t^{m}} \Phi(a t, w)=m ! \Phi_{m}(a, w)+\cdots+k(k-1) \cdot \ldots \cdot(k-m+1) t^{k-m} \Phi_{k}(a, w)+\cdots
$$

We calculate the derivative of the same as the derivative of a composite function

$$
\frac{d^{m}}{d t^{m}} \Phi(a t, w)=\sum_{\|\alpha\|=m} \frac{\partial^{\alpha} \Phi(a t, w)}{\partial z^{\alpha}} \cdot a^{\alpha} .
$$


Equating the derivatives, we obtain

$$
\sum_{\|\alpha\|=m} \frac{\partial^{\alpha} \Phi(a t, w)}{\partial z^{\alpha}} \cdot a^{\alpha}=\sum_{k=m}^{\infty} k(k-1) \cdot \ldots \cdot(k-m+1) t^{k-m} \Phi_{k}(a, w) .
$$

Substituting value $t=0$ in Eq. (13), we get that $\frac{d^{m}}{d t^{m}} \Phi(0, w)=m ! \Phi_{m}(a, w)$ is a polynomial of degree not higher than $m$ in $w$, since the left side of this equation is a polynomial of degree not higher than $m$ in $w$ by hypothesis of the theorem. When $m=0$ we get $\Phi(0, w)=\Phi_{0}(a, w)=$ const and from (11) we have $\Phi(0, w)=\Phi_{0}(a, w)=\Phi_{0}(0, w)$.

Substitute $t=1$ in Eq. (12), we obtain

$$
\Phi(a, w)=\sum_{k=0}^{\infty} \Phi_{k}(a, w)=\text { const } .
$$

Since $\Phi_{k}(a, w)=\sum_{l=k}^{\infty} P_{k, l}(a, w)$ is a polynomial in $w$ of degree not higher than $k$, then $\sum_{l=k}^{\infty} P_{k, l}(a, w)=P_{k, k}(a, w)$. Therefore

$$
\text { const }=\Phi(a, w)=\sum_{k=0}^{\infty} \Phi_{k}(a, w)=\sum_{k=0}^{\infty} P_{k, k}(a, w)
$$

Hence $P_{k, k}(a, w)=0$ for $k>0$. Therefore $\Phi_{k}(a, w)=0$ for $k>0$, so from (12) we get $\Phi(a t, w)=$ const and $\frac{\partial^{\beta} \Phi(a t, w)}{\partial w^{\beta}}=0$ at $\|\beta\|>0$.

Corollary 2. Let the function $f(\zeta) \in \mathcal{C}(S)$ have the one-dimensional holomorphic extension property along the family $\mathfrak{L}_{\{0, a\}}$, then $\Phi(z, w)=$ const for points $z$ belonging to the complex line $l_{0, a} \cap B$, i.e., $\frac{\partial^{\beta} \Phi(z, w)}{\partial w^{\beta}}=0$ at $\|\beta\|>0$.

Proof follows from Theorem 1 and Theorem 3.

Corollary 3. Under the hypotheses of Corollary 2 the equality

$$
\frac{\partial^{\beta} F(z)}{\partial \bar{z}^{\beta}}=0
$$

holds for all points $z \in l_{0, a} \cap B$ and $\|\beta\|>0$.

Theorem 4. Let the function $f(\zeta) \in \mathcal{C}(S)$ have the one-dimensional holomorphic extension property along the family $\mathfrak{L}_{\{a, c\}}, a, c \in B$, then $\Phi(a+(c-a) t, w)=$ const on $w$ for $|t|<1$, i.e., $\frac{\partial^{\beta} \Phi(a+(c-a) t, w)}{\partial w^{\beta}}=0$ at $\|\beta\|>0$.

Proof. From Theorem 2 we have

$$
\Phi(a, w)=\mathrm{const}, \Phi(c, w)=\mathrm{const} \text { and } \frac{\partial^{\alpha} \Phi(a, w)}{\partial z^{\alpha}}, \frac{\partial^{\alpha} \Phi(c, w)}{\partial z^{\alpha}}
$$

are polynomials of degree not higher than $\|\alpha\|$ in $w$. 
Expanding the function $\Phi(z, w)$ in a Taylor series at the point $(a, \bar{a})$, we obtain

$$
\Phi(z, w)=\sum_{k, l=0}^{\infty} P_{k, l}(z-a, w-\bar{a})
$$

where $P_{k, l}(z, w)$ are homogeneous holomorphic polynomials of degree of homogeneity of $k$ in $(z-a)$ and $l$ in $(w-\bar{a})$. The series (15) converges to the function $\Phi(z, w)$ in the neighborhood of $(a, \bar{a})$. We make the substitution $z-a \rightarrow z, w-\bar{a} \rightarrow w$. Then the point $c$ go to point $\tilde{c}=c-a$, and the function $\Phi(z, w)$ will pass to a function $\widetilde{\Phi}(z, w)$ holomorphic in a neighborhood $U_{z} \times U_{w}$ of zero, where $U_{z}$ is some neighborhood of zero at $z$, and $U_{w}$ is some neighborhood of zero at $w$, and the series (15) will take the form

$$
\widetilde{\Phi}(z, w)=\sum_{k, l}^{\infty} P_{k, l}(z, w)
$$

which converges in the neighborhood $U_{z} \times U_{w}$. Therefore, the conditions (14) take the form

$$
\widetilde{\Phi}(0, w)=\mathrm{const}, \widetilde{\Phi}(\tilde{c}, w)=\mathrm{const} \text { and } \frac{\partial^{\alpha} \widetilde{\Phi}(0, w)}{\partial z^{\alpha}}, \frac{\partial^{\alpha} \widetilde{\Phi}(\tilde{c}, w)}{\partial z^{\alpha}} .
$$

So they are polynomials in $w$ of degree not higher than $\|\alpha\|$. From the conditions of the theorem the expansion (16) will take the form

$$
\widetilde{\Phi}(z, w)=\sum_{k \geqslant l \geqslant 0}^{\infty} P_{k, l}(z, w),
$$

since the derivatives $\frac{\partial^{\alpha+\beta} \widetilde{\Phi}(0,0)}{\partial z^{\alpha} \partial w^{\beta}}=0$ at $\|\beta\|>\|\alpha\|$.

We introduce the functions

$$
\Phi_{k}(z, w)=\sum_{l=k}^{\infty} P_{k, l}(z, w)
$$

then

$$
\widetilde{\Phi}(z, w)=\sum_{k=0}^{\infty} \Phi_{k}(z, w) .
$$

Considered series (19) and (20) converge absolutely in $U_{z} \times U_{w}$, uniformly on compact subsets in this neighborhood, since double series (16) converges absolutely in $U_{z} \times U_{w}$ and uniformly on compact subsets in this neighborhood. Then

$$
\widetilde{\Phi}(0, w)=\Phi_{0}(0, w)=\sum_{l=0}^{\infty} P_{0, l}(0, w)=\text { const } .
$$

Consider the expansion of the function $\widetilde{\Phi}(t \tilde{c}, w)$ in a series by $\mathrm{t}$

$$
\widetilde{\Phi}(t \tilde{c}, w)=\sum_{k=0}^{\infty} t^{k} \widetilde{\Phi}_{k}(\tilde{c}, w)
$$

where $\widetilde{\Phi}_{k}(\tilde{c}, w)=\left.\frac{\partial^{k} \widetilde{\Phi}(t \tilde{c}, w)}{k ! \partial t^{k}}\right|_{t=0}$. If we apply the expansion $(20)$ to the point $(\tilde{c}, w)$, we obtain

$$
\widetilde{\Phi}_{k}(\tilde{c}, w)=\Phi_{k}(\tilde{c}, w)
$$


for sufficiently small $|w|$. We find

$$
\frac{d^{m}}{d t^{m}} \widetilde{\Phi}(t \tilde{c}, w)=m ! \widetilde{\Phi}_{m}(\tilde{c}, w)+\ldots+k(k-1) \cdot \ldots \cdot(k-m+1) t^{k-m} \widetilde{\Phi}_{k}(\tilde{c}, w)+\ldots
$$

We calculate this derivative of the same as the derivative of a composite function

$$
\frac{d^{m}}{d t^{m}} \widetilde{\Phi}(t \tilde{c}, w)=\sum_{\|\alpha\|=m} \frac{\partial^{\alpha} \widetilde{\Phi}(t \tilde{c}, w)}{\partial z^{\alpha}} \tilde{c}^{\alpha} .
$$

Equating the derivatives we obtain the equality

$$
\sum_{\|\alpha\|=m} \frac{\partial^{\alpha} \widetilde{\Phi}(t \tilde{c}, w)}{\partial z^{\alpha}} \tilde{c}^{\alpha}=\sum_{k=m}^{\infty} k(k-1) \cdot \ldots \cdot(k-m+1) t^{k-m} \widetilde{\Phi}_{k}(\tilde{c}, w) .
$$

Substituting the value $t=0$ in Eq. (23), we get that $\frac{d^{m}}{d t^{m}} \widetilde{\Phi}(0, w)=m ! \widetilde{\Phi}_{m}(\tilde{c}, w)$ is a polynomial in $w$ of degree not higher than $m$, because the left side of this equation is a polynomial in $w$ of degree not higher than $m$ from the condition (17). When $m=0$ we obtain $\widetilde{\Phi}(0, w)=\Phi_{0}(\tilde{c}, w)=$ const and from (21) we have $\widetilde{\Phi}(0, w)=\widetilde{\Phi}_{0}(\tilde{c}, w)=\Phi_{0}(0, w)$.

From Eqs. (19) and (22) we find that $\widetilde{\Phi}_{k}(\tilde{c}, w)=P_{k, k}(\tilde{c}, w)$ for sufficiently small $|w|$, i.e., $\widetilde{\Phi}_{k}(\tilde{c}, w)$ is a polynomial of degree exactly $k$ in $w$. Therefore

$$
\text { const }=\widetilde{\Phi}(\tilde{c}, w)=\sum_{k=0}^{\infty} P_{k, k}(\tilde{c}, w)
$$

Hence $P_{k, k}(\tilde{c}, w)=0$ at $k>0$. Here $\widetilde{\Phi}(\tilde{c}, w)=0$ at $k>0$, therefore from (21) we get that $\widetilde{\Phi}(t \tilde{c}, w)=$ const, i.e., $\frac{\partial^{\beta} \widetilde{\Phi}(t \tilde{c}, w)}{\partial w^{\beta}}=0$ at $\|\beta\|>0$.

Corollary 4. Under the hypotheses of Theorem 4 the equality $\left.\frac{\partial^{\beta} F(z)}{\partial \bar{z}^{\beta}}\right|_{z=a+(c-a) t}=0$ holds at $\|\beta\|>0$.

\section{Main results}

Theorem 5. Let $n=2$ and the function $f(\zeta) \in \mathcal{C}(S)$ have the one-dimensional holomorphic extension property along the family $\mathfrak{L}_{\{a, c, d\}}$, points $a, c, d \in B$ do not lie on one complex line in $\mathbb{C}^{2}$, then $\frac{\partial^{\beta} \Phi(z, w)}{\partial w^{\beta}}=0$ for any $z \in B$ and $\|\beta\|>0$, i.e., $F(z)$ is holomorphic in $B$ (then $f(\zeta)$ extends holomorphically into $B$ ).

Proof. Let $\tilde{z}$ be an arbitrary point on the line $l_{a, c}$, then by the Theorem 4 we have

$$
\frac{\partial^{\beta} \Phi(\tilde{z}, w)}{\partial w^{\beta}}=0
$$

at $\|\beta\|>0$. We connect the point $\tilde{z}$ to the point $d$ of the line $l_{\tilde{z}, d}$ and again apply Theorem 4 for the points $\tilde{\tilde{z}} \in l_{\tilde{z}, d}$, we get that $\frac{\partial^{\beta} \Phi(\tilde{\tilde{z}}, w)}{\partial w^{\beta}}=0$ at $\|\beta\|>0$. Therefore, the condition (24) is satisfied for all points $\tilde{z}$ from some open set. 
Substituting in Eq. (24) $w=\bar{z}$ and using Eq. (5), we find that $\frac{\partial^{\beta} F(z)}{\partial \bar{z}^{\beta}}=0$ in an open set of the ball $B$. Due to the real-analytic of function $F(z)$, it follows that $\frac{\partial F(z)}{\partial \bar{z}_{j}}=0$ for any $z \in B$ and $j=1, \ldots, n$. Since $\left.F(z)\right|_{\partial B}=f(\zeta)$, then the function $f(\zeta)$ extends holomorphically to the ball $B$.

From Theorem 5 we get that in the ball $B \subset \mathbb{C}^{2}$ a sufficient set for a continuous function defined on the boundary of the ball is the set $\mathfrak{L}_{\{a, c, d\}}$, where $a, c, d$ are arbitrary points of the ball, not lying on one complex line.

We denote $\mathcal{A}$ the set of points $a_{k} \in B \subset \mathbb{C}^{n}, k=1, \ldots, n+1$, do not lie on complex hyperplane in $\mathbb{C}^{n}$.

Theorem 6. Let $f(\zeta) \in \mathcal{C}(S)$ have the one-dimensional holomorphic extension property along the family $\mathfrak{L}_{\mathcal{A}}$, then $\frac{\partial^{\beta} \Phi(z, w)}{\partial w^{\beta}}=0$ for any $z \in B$, i.e., $F(z)$ is holomorphic in $B$ (then $f(\zeta)$ extends holomorphically into $B$ ).

Proof. The proof goes by induction on $n$. The basis of induction is Theorem $5(n=2)$. Suppose that for all dimensions $k<n$ theorem is true. Without loss of generality when $k=n$, we assume that $a_{n+1}=0$.

Consider the complex plane $\Gamma$, passing through the points $a_{1}, \ldots, a_{n}$, its dimension, by assumption, is $n-1$ and $0 \notin \Gamma$. The intersection of $\Gamma \cap B$ is some ball in $\mathbb{C}^{n-1}$. The function $\left.f\right|_{\Gamma \cap S}$ is continuous and has the property of holomorphic extension along the family $\mathfrak{L}_{\mathcal{A}_{1}}$, where $\mathcal{A}_{1}=\left\{a_{1}, \ldots, a_{n}\right\}$. By the induction assumption $\frac{\partial^{\beta} \Phi\left(z^{\prime}, w\right)}{\partial w^{\beta}}=0$ at $\|\beta\|>0$ for all $z^{\prime} \in \Gamma \cap B$.

Connecting points $z^{\prime} \in \Gamma$ with the point 0 we get by Theorem 3 , that $\frac{\partial^{\beta} \Phi(z, w)}{\partial w^{\beta}}=0$ at $\|\beta\|>0$ for some open set in $B$. Hence, as in Theorem 5 , we see that $F(z)$ is holomorphic in $B$.

Corollary 5. Under the hypotheses of Theorem 6 the equality $\frac{\partial^{\beta} F(z)}{\partial \bar{z}^{\beta}}=0$ holds for any $z \in B$ and $\|\beta\|>0$ and $f(\zeta)$ extends holomorphically into $B$.

The authors used the financial support of RFBR, grant 14-01-00544.

\section{References}

[1] M.L.Agranovskii, R.E.Valskii, Maximality of invariant algebras of functions, Sib. Math. J., 12(1971), no. 1, 1-7.

[2] E.L.Stout, The boundary values of holomorphic functions of several complex variables, Duke Math. J., 44(1977), no. 1, 105-108.

[3] L.A.Aizenberg, A.P.Yuzhakov, Integral representations and residues in multidimensional complex analysis, Amer. Mat. Soc., Providence, 1981.

[4] A.M.Kytmanov, S.G.Myslivets, Higher-dimensional boundary analogs of the Morera theorem in problems of analytic continuation of functions, J. Math. Sci., 120(2004), no.6, $1842-1867$.

[5] J.Globevnik, E.L.Stout, Boundary Morera theorems for holomorphic functions of several complex variables, Duke Math. J., 64(1991), no. 3, 571-615. 
[6] A.M.Kytmanov, S.G.Myslivets, On the families of complex lines, sufficient for holomorphic continuation, Mat. Notes., 83(2008), no. 4, 545-551.

[7] A.M.Kytmanov, S.G.Myslivets, V.I.Kuzovatov, Families of complex lines of the minimal dimension, sufficient for holomorphic continuation of functions, Sib. Math. J., 52(2011), no. 2, 256-266.

[8] M.Agranovsky, Propagation of boundary $C R$-foliations and Morera type theorems for manifolds with attached analytic discs, Advances in Math., 211(2007), no. 1, 284-326.

[9] M.Agranovsky, Analog of a theorem of Forelli for boundary values of holomorphic functions on the unit ball of $\mathbb{C}^{n}$, Journal d'Analyse Mathematique, 13(2011), no. 1, 293-304.

[10] L.Baracco, Holomorphic extension from the sphere to the ball, Journal of Mathematical Analysis and Applications, 2012(388), no. 2, 760-762.

[11] J.Globevnik, Small families of complex lines for testing holomorphic extendibility, Amer. J. of Math., 134(2012), 1473-1490.

[12] L.Baracco, Separate holomorphic extension along lines and holomorphic extension from the sphere to the ball, Amer. J. of Math., 135(2013), 493-497.

[13] J.Globevnik, Meromorphic extensions from small families of circles and holomorphic extensions from spheres, Trans. Amer. Math. Soc., 364(2012), 5857-5880.

[14] A.M.Kytmanov, S.G.Myslivets, Holomorphic Continuation of Functions Along Finite Families of Complex Lines in the Ball, Journal of Siberian Federal University. Mathematics \& Physics, 5(2012), 547-557 (in Russian).

[15] A.M.Kytmanov, S.G.Myslivets, An Analogue of the Hartogs Theorem in a Ball of $\mathbb{C}^{n}$, Mathematische Nachrichten, 288(2015), 224-234.

[16] A.M.Kytmanov, The Bochner-Martinelli integral and its applications, Birkhäuser, Basel, Boston, Berlin, 1995.

[17] W.Rudin, Function Theory in the Unit Ball of $\mathbb{C}^{n}$, Springer-Verlag, Berlin, Heidelberg, New-York, 1980.

\section{Голоморфное продолжение непрерывных функций вдоль конечных семейств комплексных прямых в шаре}

\section{Александр М. Кытманов Симона Г. Мысливец}

\footnotetext{
B этой статъе мы рассматриваем непрерывные функции, заданные на границе шара $B$ в $\mathbb{C}^{n}$, $n>1$, и обладающие одномерным свойством голоморфного продолжения вдоль семейств комплексных прямых, проходящих через конечное число точек из В. Мы доказываем, что это семейство является достаточнымдля голоморфного продолжения таких функций в шар $B$.
}

Ключевые слова: голоморфное продолжение, ядро Пуассона, комплексные прямые. 